\section{The anxiety-reducing effects of cannabidiol treatment in teenagers with social anxiety disorder}

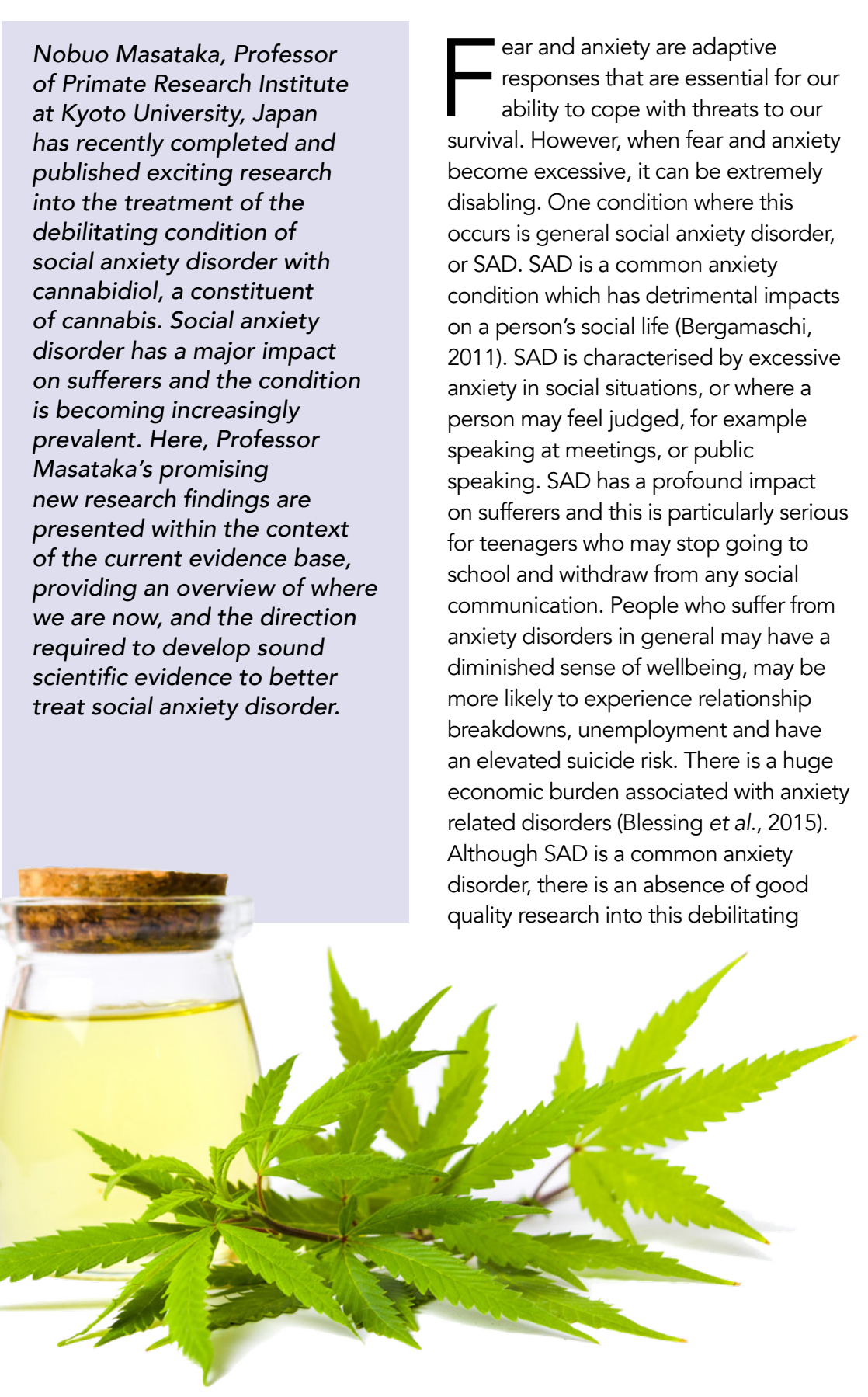

condition, meaning that the cause of SAD nd the treatment of the disorder are not fully understood.

The treatment of SAD with currently vailable medications is problematic with only around $30 \%$ of patients showing a recovery without ongoing symptoms (Blanco et al., 2002). The current range of pharmacological medications are limited by adverse side effects. Blessing and colleagues note the high priority to develop novel alternative medications for the benefit of patients and to relieve the substantial burden on society.

CANNABIDIOL AS

A TREATMENT OPTION

Cannabidiol, otherwise known as CBD, is Cotention potentialin the treatment of chronic pain, 2019). Additions aly CBD hos properties hat reverse anxiety-like behaviour in humans and animals and as such has been regarded as a potential treatment option for SAD.

Recreational and medicinal users of illegally available cannabis have reported side effects of panic and anxiety. One constituent of cannabis, D9-tetrahydrocannabinol (THC), causes the euphoria and mind-altering effects of cannabis, but detrimentally, THC has been proven to increase anxiety levels in cannabis users. CBD, which acts very differently on the body to THC, does not have the psychoactive efrects of THC, induce an anti-anxiety effect and works to reduce anxiety.

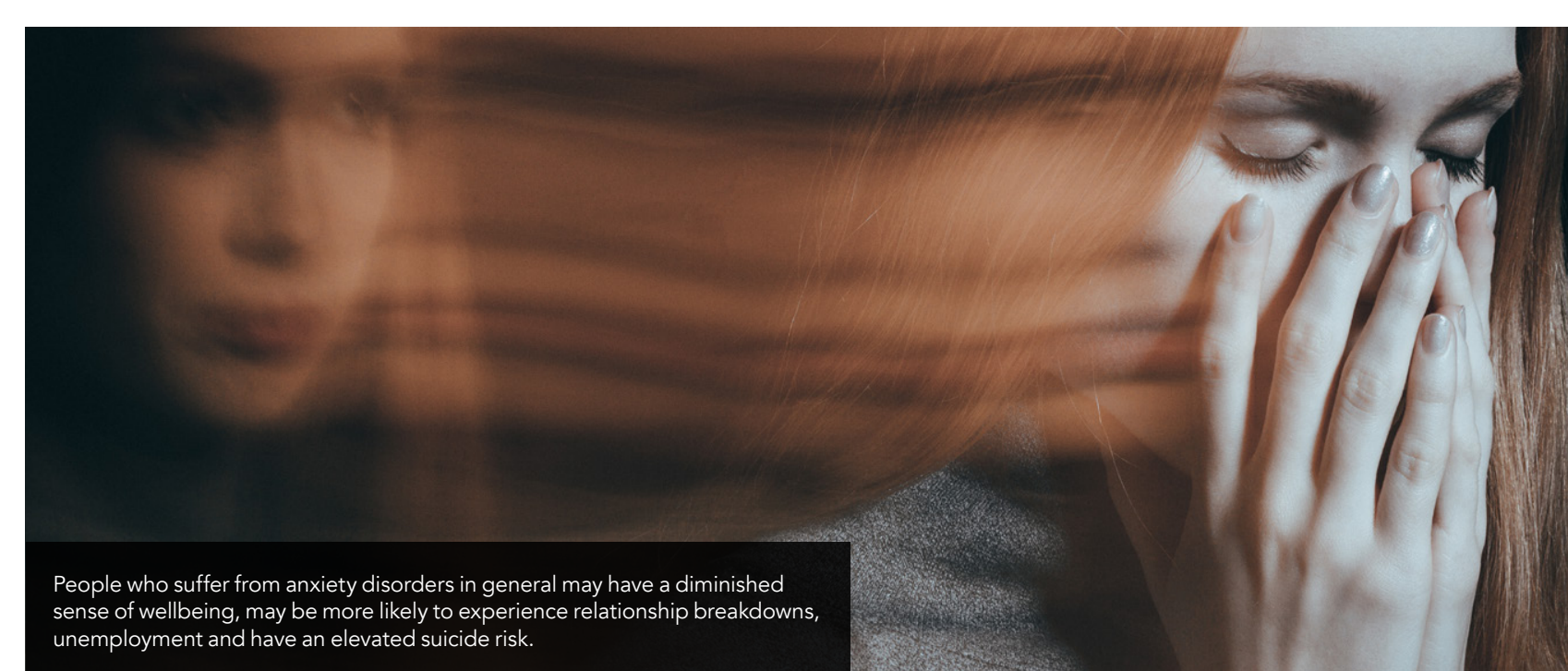

In relation to $S A D$, the benefits of CBD over alternative medications is have the side effects assd does not medications traditionaly used to treat SAD. Bergamaschi and colleagues report that up to 1500 of CBD mg day is well tolerated by patients with no reports of slowed responses, poor mood, nor any detrimental impacts. on the heart and breathing rates. One of the current medication options for SAD might be benzodiazepines. Unlike treatment with CBD, benzodiazepines may impair function, cause dependence and withdrawal symptoms

EXISTING EVIDENCE

UPPORTING CANNABIDIOL (lessing and colleagues published existing evidence for the use of CBD to treat anxiety-related disorders. It appraised the evidence from a broad range of studies to include preclinical evidence, human experimental studies and epidemiological studies (Blessing et a). The findings indicated that animal studies support the use of CBD to treat anxiety related disorders. The team also found that human studies are supportive of the anti-anxiety effects of CBD, but there was a need for further research in this area to support the use of CBD as a treatment option for specific anxiety related conditions, and also to investigate which are the optimum levels of dosing.

A small trial investigating the effects of CBD on participants with SAD who were
public speaking (which induces similar anxiety effects to SAD), found that those people who were treated with CBD had when they were speaking (Bergan et al. The authors recognised the need for a large trial to further test the effects of CBD on anxiety.

CANNABIDIOL TO TREAT SAD: NEW FINDINGS

Professor Masataka and colleagues have recently published their exciting clinical trial investigating the effects of CBD on teenagers experiencing SAD in Frontiers in Psychology. This trial shows promising results concerning the treatment of $S A$ with CBD. Masataka and colleagues

SAD who were randomly allocated to receive treatment with either $C B D$, or placebo treatment contrinent. The the CBD treatment contained 300mg of CBD oil. The treatment was administered by a clinical psychologist, unaware of the teenagers' treatment allocation, at the participants' home. Anxiety was measured before and after treatment using the Fear of Negative Evaluation Questionnaire and the Liebowitz Social Anxiety Scale. The teenage participants were followed up for 6 months after their treatment for brief health checks. In this trial neither the researchers nor the teenage participants were aware whether

SAD is a common anxiety condition which has detrimental impacts on a person's social life.

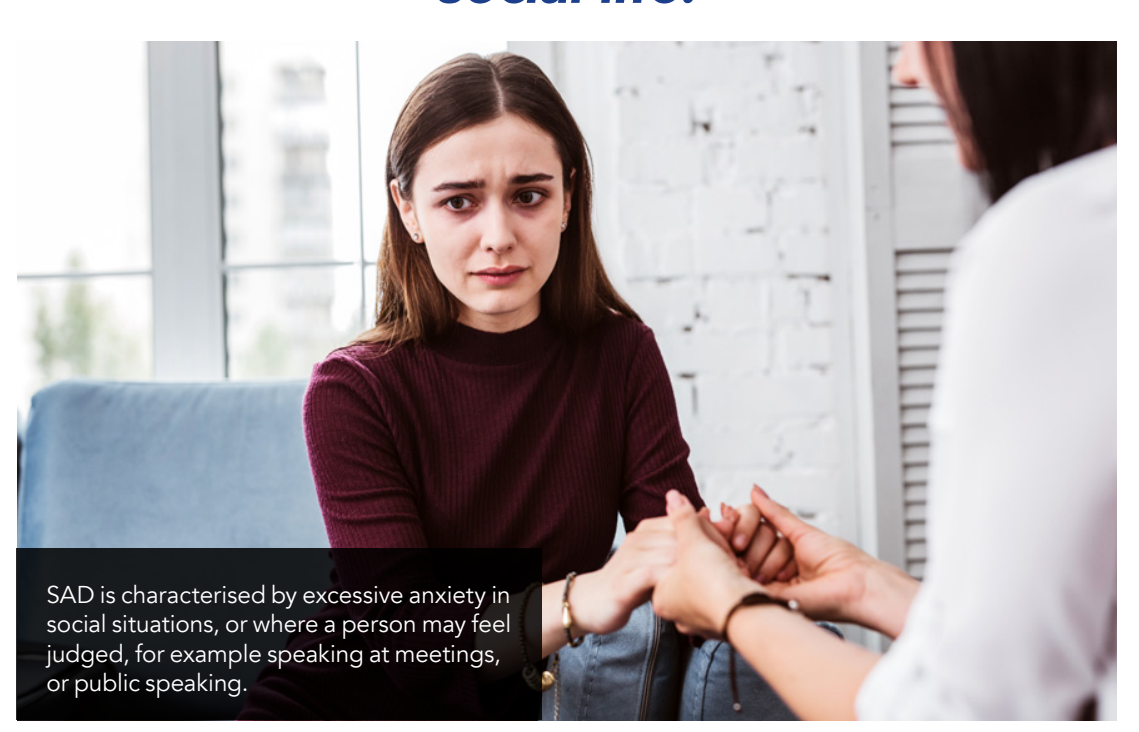




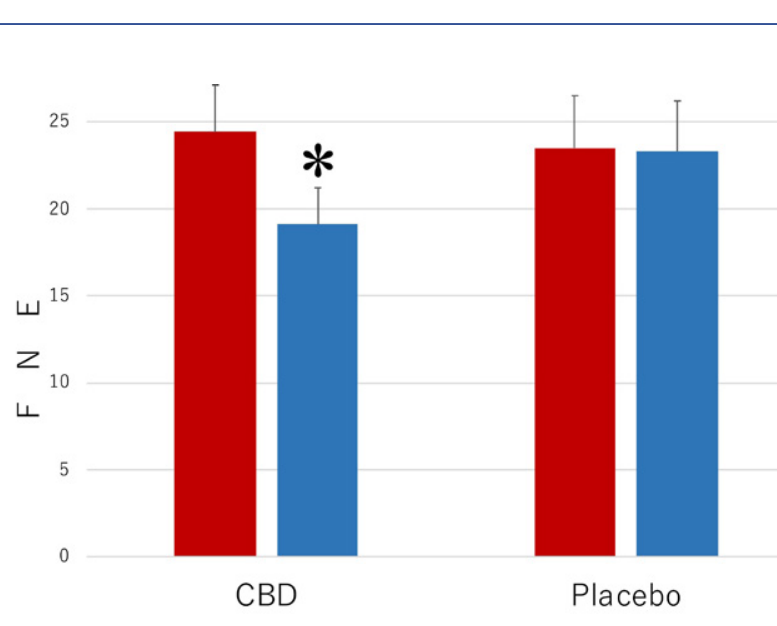

Fig 1. Scores of Fear of Negative Evaluation Questionnaire (FNE) in the
particicipants who receeived CBD and in the participants who received placebo. The participants were evaluated before and after treatment Error bars represent SDs. ${ }^{*}$.
pretreatment measurement.

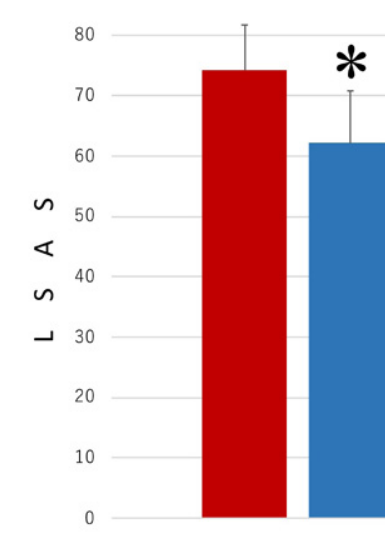

Fig 2. Scores of Liebowitz Social Anxiety Scale (LSAS) in the
participants who received CBD and in the participants whe

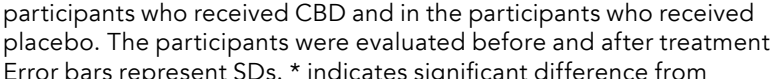
Error bars represent SDs."
Masataka and colleagues' trial revealed exciting findings. The results present effects of $C B D$ on teenagers suffering with SAD. The teenagers who received the treatment with CBD had reduced anxiety when compared with the teenagers who received the placebo treatment. Although this study did not plan to specifically record any side effects of treatment, none of the teenagers who participated had any significant health complaints following the trial. An additional finding of

interest was that $53 \%$ of the teenagers
who received treatment with CBD oil

went on to take the decision to receive treatment (medication and Cognitive Behavioural Therapy) from a hospita placebo group sought any additional treatment. This finding means that the patients who received CBD felt the capacity to overcome their anxieties concerning the stigma of their condition and engaging with therapists, to access existing treatments. The fact that teenagers suffering with SAD who were treated with $\mathrm{CBD}$ were making positive steps towards ongoing treatment is a of note.

The results present the first clinical evidence of the positive effects of CBD on teenagers suffering with SAD.

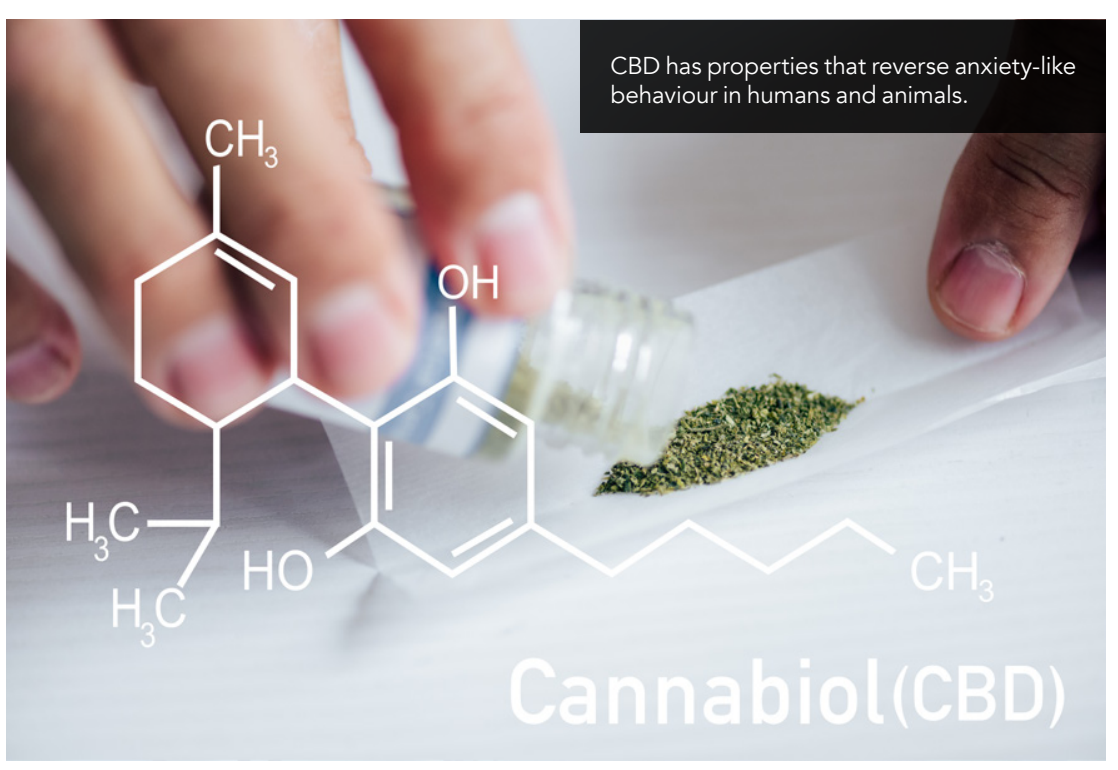

\section{SONG FORWARD}

Masataka and colleagues note that shown to be one of the most coeen psychiatric disorders. Symptoms may begin as early as five years old and peak the age of twelve Left untreated the disorder can continue into adolescence and then into adulthood. In Japan thas been reported that there are a million teenagers with SAD who may withdraw into their living spaces for up to six months at a time. Professor Masataka is highly encouraged by the results of his trial of CBD in teenagers with SAD. Indeed, he is hopeful that the results of his present study will contribute to the development of sound scientific evidence and the development of further human clinic trials to advance the scientifically proper use of CBD in patients who are recommends a further clinical trial of robust design, which compares treatment of SAD with CBD and the most commonly used pharmacological treatment option. A well-designed trial of this nature could provide the sound scientific evidence base to further support the safe and effective use of CBD in the treatment of SAD. The potential benefits of treatment with CBD for SAD are evident. Robust clinical trials are clearly needed to identify a definitive scientific evidence base to support treatment of SAD with CBD. In addition, there is a need to identify the most appropriate dosages debilitating of conditions, affect many people worldwide.

\section{Research Objectives}

obuo Masataka's research interests include origins and evolution of languages as well as developmental disorders. He is also interested in exploring interventions for aut

\section{Detail}

Nobuo Masataka

Institute

Kyoto University
Inuyama 484-8506

Japan

Nobuo Masataka obtained his PhD from Osaka University in the ethological study of vocal

communication in New World primates. As Assistant Professor of Kyoto University and of the University of Tokyo, he has published numerous articles about language learning of preverbal infants as well as a book "The Onset of Language" from Cambridge University Press. Dr Masataka currently holds a Professorship the Primate Research Institute, Kyoto University.

\section{Funding}

FPS research grant \#125285201

Collaborators

Satomi Yamada

- Atsushi Ishige

- Ryohei Tatsum

- Koji Maki

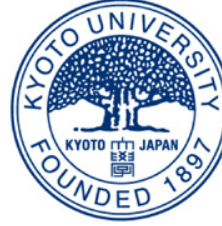

京都大学

KYOTO UNIVERSITY

\section{References}

Bergamaschi, M., et al. (2011). Cannabidiol reduces the anxiety induced by simulated public speaking in treatmentnaive social phobia patients. Neuropsychophar macology. 36(6), 1219 - 1226. doi: 10.1038/npp.2011.6

Bergamaschi M., Costa Queiroz R., Zuardi A., \& Crippa, A. (2011). Safety and side effects of cannabidiol, a Cannab doi:102174/157488611798280924

Blanco C. Antia S. Liebowitz M. (2002). Pharmacotherapy of Social Anxiety Disorder. Biol Psychiatry 51: 109-120.

Blessing, E., et al. (2015). Cannabidiol as a potential treatment for anxiety disorders. Neurotherapeutics, 12(4) 825-836. https

Liebowitz M. (1987). Social Phobia. Mod Probl Pharmacopsychiatry. 22, 141-173

Masataka, N. (2019). Anxiolytic Effects of Repeated Cannabidiol Treatment in Teenagers with Social Anxiety Disorders. Frontiers in Psychology, 10. https://doi. org/10.3389/fpsyg.2019.02466.

\section{Personal Response}

Social anxiety disorder (SAD) is becoming increasingly common. How do you envisage the research into the use of cannabidiol developing and do you foresee that cannabidiol will become experience SAD?

II What is needed as a next step to introduce CBD for CBD is appropriate daily, according to the severity of the disorder that each person experiences. Ideally, I hope CBD should not only used as a medicine, but as a supplement. My sense is that the number of people living with socia phobia is thely much higher than what is assumed. A university or started a new job) may experience difficulty in communicating with others and somehow become phobic socially. Under such circumstances, the use of CBD would prevent them from growing worse and help restoring their
mental health. 